\title{
Design of the Shunyi study on cardiovascular disease and age-related brain changes: a community-based, prospective, cohort study
}

\author{
Fei Han ${ }^{1 \#}$, Li-Xin Zhou ${ }^{1 \#}$, Jun Ni ${ }^{1}$, Ming Yao ${ }^{1}$, Fei-Fei Zhai ${ }^{1}$, Yong-Tai Liu ${ }^{2}$, Wei Wu ${ }^{2}$, Hua-Dan Xue ${ }^{3}$, \\ Ming-Li Li ${ }^{3}$, Meng Yang ${ }^{4}$, Qing Dai ${ }^{4}$, Li-Ying Cui ${ }^{1}$, Zheng-Yu Jin ${ }^{3}$, Yi-Cheng Zhu ${ }^{1 \dagger}$, Shu-Yang Zhang ${ }^{2 \dagger}$ \\ ${ }^{1}$ Department of Neurology, Peking Union Medical College Hospital, Chinese Academy of Medical Sciences, Beijing, China; ${ }^{2}$ Department of \\ Cardiology, Peking Union Medical College Hospital, Chinese Academy of Medical Sciences, Beijing, China; ${ }^{3}$ Department of Radiology, Peking \\ Union Medical College Hospital, Chinese Academy of Medical Sciences, Beijing, China; ${ }^{4}$ Department of Ultrasound, Peking Union Medical College \\ Hospital, Chinese Academy of Medical Sciences, Beijing, China \\ Contributions: (I) Conception and design: YC Zhu, SY Zhang; (II) Administrative support: YC Zhu, SY Zhang; (III) Provision of study materials \\ or patients: YC Zhu, LX Zhou; (IV) Collection and assembly of data: F Han, LX Zhou, J Ni, M Yao, FF Zhai, YT Liu, W Wu, HD Xue, ML Li, \\ M Yang; (V) Data analysis and interpretation: F Han, LX Zhou, Q Dai, LY Cui, ZY Jin, YC Zhu; (VI) Manuscript writing: All authors; (VII) Final \\ approval of manuscript: All authors. \\ \#These authors contributed equally to this work. \\ ${ }^{\dagger}$ These authors contributed equally to this work. \\ Correspondence to: Shu-Yang Zhang. Department of Cardiology, Peking Union Medical College Hospital, Peking Union Medical College and \\ Chinese Academy of Medical Science, No. 1 Shuaifuyuan, Wangfujing, Beijing 100730, China. Email: shuyangzhang103@163.com; Yi-Cheng Zhu. \\ Department of Neurology, Peking Union Medical College Hospital, Peking Union Medical College and Chinese Academy of Medical Science, No. \\ 1 Shuaifuyuan, Wangfujing, Beijing 100730, China. Email: zhuych910@163.com.
}

\begin{abstract}
Background: Rapid economic growth and increasing lifespan have contributed to an increasing burden of chronic non-communicable diseases in China. Population-based studies focusing on cardiovascular disease and age-related brain changes, with extensive clinical, genetic, and imaging data as well as a comprehensive evaluation of brain function are lacking in China. The Shunyi cohort study aimed to investigate the determinants and consequences of cardiovascular disease and age-related brain alterations among individuals residing in a rural area of Beijing.

Methods: This prospective, community-based study included individuals aged 35 years and older living in five villages in Shunyi, a rural district located 20 miles from urban Beijing. A total of 1,586 individuals were enrolled between June 2013 and September 2014. Biological samples and brain magnetic resonance images were collected along with baseline clinical data through face-to-face interviews. Whole exome sequencing and quantitative assessments of cognitive and motor function were performed.

Results: Among the 1,586 participants included, 40\% were men, and the mean age of the cohort was $56.7 \pm 10.0$ years. This population had a relatively low education level. A heavy burden of vascular risk factors with a low control rate was observed in the Shunyi population. Since 2017, the cohort has been followed up annually. As of October 2019, we had failed to obtain the follow-up data of five participants.

Conclusions: With an extensive range of clinical, genetic, and imaging data, the Shunyi cohort study has the potential to contribute significantly towards identifying the causes and consequences of cardiovascular disease and age-related brain changes in older people in China.
\end{abstract}

Keywords: Community-based; China; cardiovascular disease; brain structure; brain function

Submitted May 23, 2020. Accepted for publication Oct 10, 2020.

doi: 10.21037/atm-20-4195

View this article at: http://dx.doi.org/10.21037/atm-20-4195 


\section{Introduction}

China has experienced rapid economic growth and its citizens have seen a significant transformation in their lifestyles over the past few decades. This has been accompanied by an epidemiological shift from infectious diseases to chronic non-communicable diseases $(1,2)$. The population age structure is also shifting due to increased fertility and declining mortality rates, thereby increasing the proportion of older people. The heavy burden of cardiovascular diseases and neurological disorders driven by urbanization, behavioral transition, increasing incomes, aging, and longer lifespan poses great challenges for China's health system (1-3).

Both environmental and hereditary factors may impact cardiovascular disease and brain health in the aging population (4-7). However, in China, studies in the general population to date have focused mostly on hypertension, diabetes, and cardiovascular disease (8); they have not assessed the contribution of environmental and genetic factors to brain health in the aging population. The sociocultural and lifestyle factors in China significantly differ from those of Western countries; moreover, China has the largest population of a single race in the world. Thus, in light of other worldwide community-based studies, a cohort study in a Chinese population with in-depth evaluations of multiple organs and genetic information is indispensable.

The Shunyi study was a community-based, prospective cohort study that enrolled participants from a rural area of Beijing, China. Clinical, biological, and imaging data were collected, with emphasis on the evaluation of the cardiovascular and neurological systems. This study aimed to investigate the environmental and hereditary determinants and consequences of cardiovascular diseases and age-related brain disorders. We hope that the study findings will help to develop practical strategies for the prevention and management of these chronic diseases in older people in China.

We present the following article in accordance with the STROBE reporting checklist (available at http://dx.doi. org/10.21037/atm-20-4195).

\section{Methods}

\section{Study design and participants}

The Shunyi cohort study is a prospective community-based study, being conducted in Shunyi, a rural district located 20 miles from Beijing's urban area (Figure 1). There are
12 towns and 428 villages in the Shunyi district. Among them, five villages willing to collaborate were selected. These selected villages have a low floating population and a demographic profile similar to that of the other villages in Shunyi.

Participant inclusion criteria were as follows: (I) local permanent residents aged 35 years or older living independently; (II) availability for long-term follow-up; (III) willingness to provide voluntary informed consent. The exclusion criteria were as follows: (I) unwillingness to participate in the study and (II) physical or mental incapability to complete the questionnaire interviews and examinations.

Extensive publicity campaigns were launched, which included the dissemination of information using banners and posters, and promotions through radio programs. Invitation letters were delivered door-to-door to the eligible residents by community social workers. From June 2013 to September 2014, among the 2,237 eligible residents, 1,586 individuals participated (response rate, $70.9 \%$ ), and standard baseline assessments were conducted (Figure 2). Emigration outside the village for employment was the primary reason for not participating in the study. This study was conducted in accordance with the Declaration of Helsinki (as revised in 2013). The study was approved by the Ethical Committee of Peking Union Medical College Hospital (No: B-160), and informed consent was obtained from all participants.

\section{Data collection}

Face-to-face interviews, physical examinations, blood sample collection, and cognitive and motor function evaluations were conducted at public activity centers of the village committees. Cardiovascular assessments, including ultrasonography of the carotid arteries, electrocardiogram, transthoracic echocardiography, and measurement of brachial-ankle pulse wave velocity (baPWV), were performed on the same day as the interview. Magnetic resonance imaging (MRI) was carried out from 2014 to 2016 in Shunyi Hospital, located in downtown Shunyi.

\section{Demographic and clinical parameters}

Standardized questionnaire-based surveys were conducted via face-to-face interviews by trained interviewers. The questionnaires included questions on demographic characteristics, socioeconomic status, lifestyle habits (physical exercise, diet habits, smoking, and alcohol 


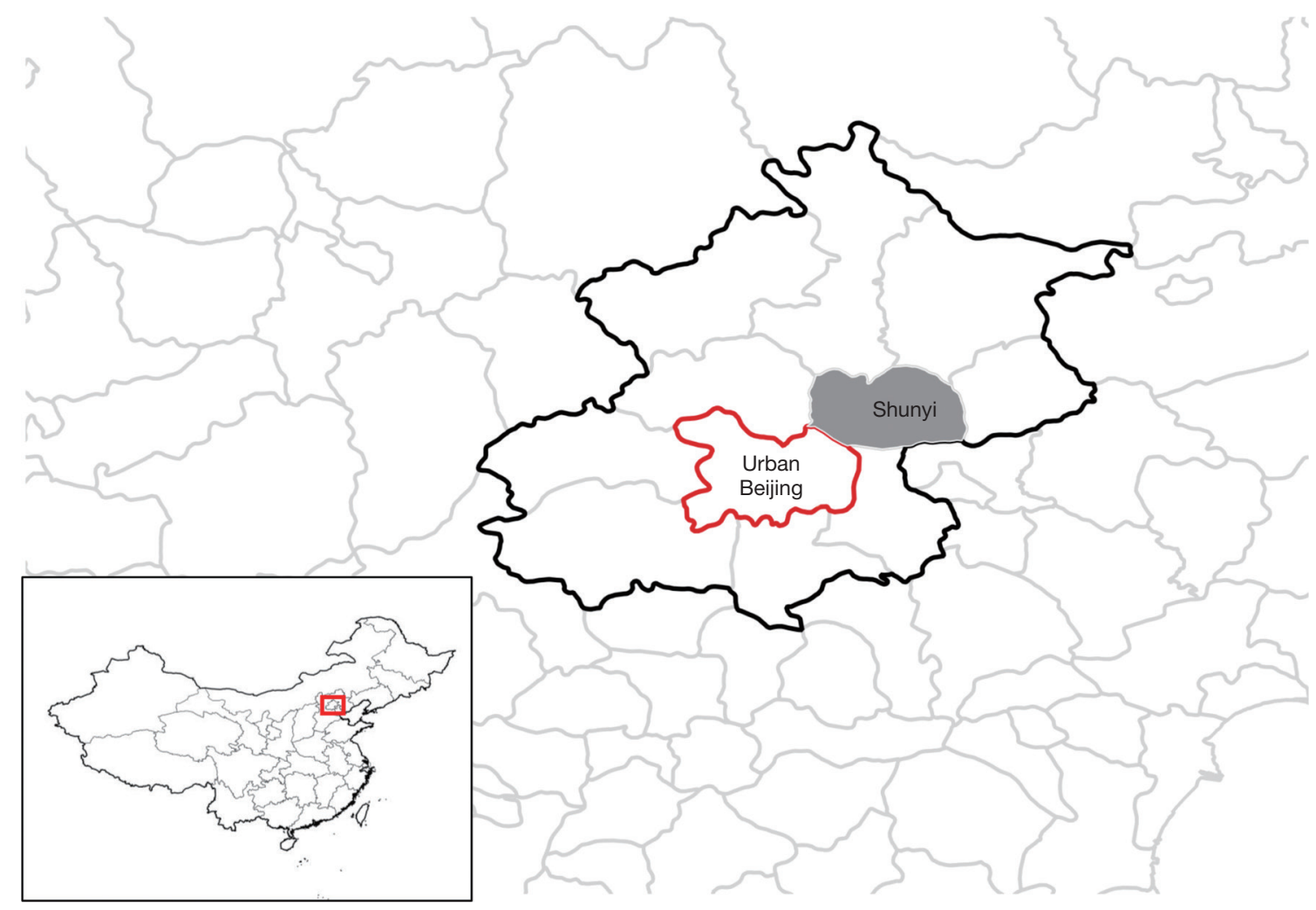

Figure 1 Location of the Shunyi district in China.

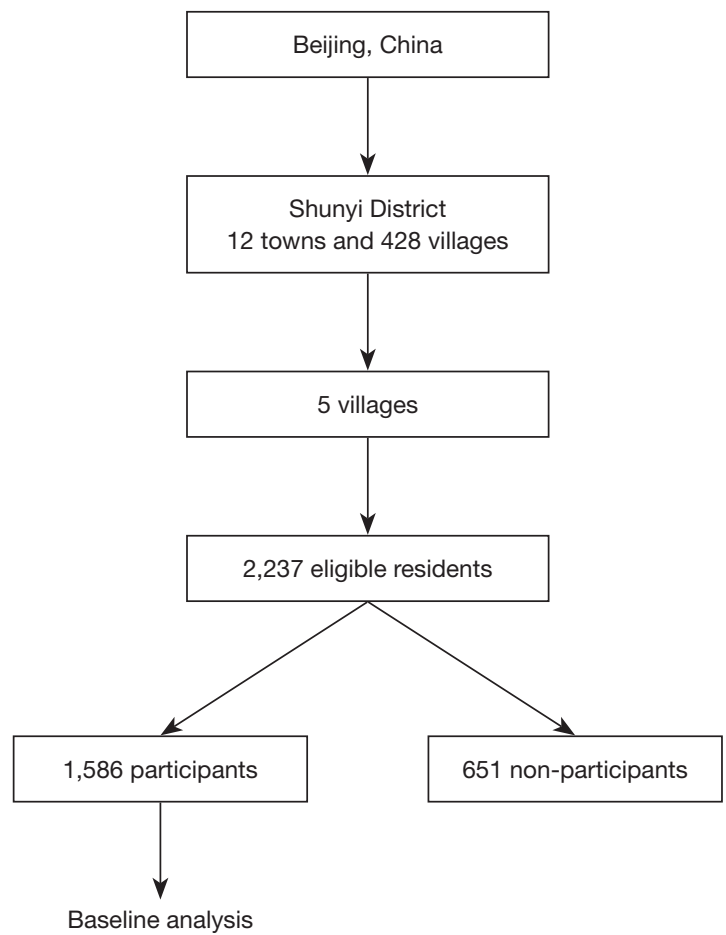

Figure 2 Flowchart of participant recruitment. consumption), medical history, current medication use, and family history. Participants then underwent a range of physical assessment measures, including anthropometric and detailed neurological examinations. Blood pressure was measured three times, and the mean values were used for analysis.

\section{Biological data and blood bank specimens}

Venous blood and urine samples were obtained after overnight fasting. Measurements of biological parameters, such as serum glucose, lipid profiles, homocysteine, C-reactive protein, plasma/urine albumin, and creatinine, were performed at central laboratories. Blood bank specimens [serum, plasma, free circulating nucleic acids, ribonucleic acid, deoxyribonucleic acid (DNA), and peripheral blood mononuclear cells] were subsequently stored for future analysis in a $-80{ }^{\circ} \mathrm{C}$ freezer, or in liquid nitrogen tanks. Genomic DNA extracted from the peripheral blood mononuclear cells of each sample was fragmented to an average size of $180-280 \mathrm{bp}$ and used to create a DNA library according to standard protocols. 
Table 1 Parameters of magnetic resonance imaging sequences

\begin{tabular}{|c|c|}
\hline Sequences & Parameters \\
\hline $\mathrm{T} 2 \mathrm{Wl}$ & $\mathrm{TR}=6,000 \mathrm{~ms}, \mathrm{TE}=125 \mathrm{~ms}, \mathrm{FOV}=229 \times 229 \mathrm{~mm}^{2}$, slice thickness $=5 \mathrm{~mm}$, gap $=1 \mathrm{~mm}, 20$ axial slices \\
\hline FLAIR & $\mathrm{TR}=8,500 \mathrm{~ms}, \mathrm{TE}=81 \mathrm{~ms}, \mathrm{FOV}=230 \times 230 \mathrm{~mm}^{2}$, slice thickness $=5 \mathrm{~mm}$, gap $=1 \mathrm{~mm}, 20$ axial slices \\
\hline SWI & $\mathrm{TR}=8,500 \mathrm{~ms}, \mathrm{TE}=81 \mathrm{~ms}, \mathrm{FOV}=230 \times 230 \mathrm{~mm}^{2}$, slice thickness $=5 \mathrm{~mm}$, gap $=1 \mathrm{~mm}, 20$ axial slices \\
\hline TOF-MRA & TR $=21 \mathrm{~ms}, \mathrm{TE}=3.43 \mathrm{~ms}, \mathrm{FOV}=208 \times 229 \mathrm{~mm}^{2}$, voxel size $=0.3 \times 0.3 \times 0.6 \mathrm{~mm}^{3}$, flip angle $=18^{\circ}, 136$ axial slices \\
\hline HR-MRI & $\mathrm{TR}=700 \mathrm{~ms}, \mathrm{TE}=22 \mathrm{~ms}, \mathrm{FOV}=184 \times 190 \mathrm{~mm}^{2}$, flip angle $=120^{\circ}$, slice thickness $=0.8 \mathrm{~mm}, 96$ coronal slices \\
\hline T1-Dixon & $\mathrm{TR}=5.36 \mathrm{~ms}, \mathrm{TE}=1.24 \mathrm{~ms}, \mathrm{FOV}=500 \times 100 \mathrm{~mm}^{2}$, flip angle $=9^{\circ}$, slice thickness $=5 \mathrm{~mm}$ without gap, $40-56$ coronal slices \\
\hline
\end{tabular}

3DT1WI, three-dimensional T1-weighted images; T2WI, T2-weighted images; FLAIR, fluid-attenuated inversion recovery; SWI, susceptibility-weighted sequences; DTI, diffusion tensor images; TOF-MRA, time-of-flight magnetic resonance angiography; HR-MRI, high-resolution magnetic resonance imaging; TR, repetition time; TE, echo time; FOV, field of view.

The Agilent SureSelect Human All ExonV6 Kit (Agilent Technologies, Santa Clara, CA, USA) was used for exome capture. The Illumina Novaseq 6000 platform (Illumina Inc., San Diego, CA, USA) was used for genomic DNA sequencing by Novogene Bioinformatics Technology Co., Ltd. (Beijing, China) to generate 150-bp pairedend reads with a mean coverage of more than $100 \times$ and a minimum coverage of $10 \times$ for $\sim 98 \%$ of the target regions of each sample. In addition, the participants were also genotyped using the Illumina Asian Screening Array (ASA) BeadChip, designed by Capitalbio Technology Co., Ltd. (Beijing, China) for detecting 698,500 single-nucleotide polymorphisms.

\section{MRI examination}

A single 3T Skyra scanner (Siemens, Erlangen, Germany) was used to obtain three-dimensional T1-weighted, $\mathrm{T} 2$-weighted, fluid-attenuated inversion recovery, and susceptibility-weighted sequences. Diffusion tensor images were acquired using a single-shot echo-planar imaging sequence covering the whole brain. Three-dimensional time-of-flight magnetic resonance angiography was performed in the axial plane. Three-dimensional T1weighted sequences of high-resolution MRI were obtained in coronal planes to analyze intracranial atherosclerotic plaques. Whole-body T1 Dixon images were acquired in the coronal plane. The parameters of each MRI sequence are summarized in Table 1.

\section{Ultrasonography of the carotid arteries}

The presence of carotid plaque was determined in the bilateral common, internal, and bifurcation sites of the carotid arteries with a color Doppler ultrasound diagnostic system (Esaote, Firenze, Italy), using a $5-13 \mathrm{MHz}$ vascular probe LA523 according to a standardized scanning protocol. The common carotid arterial (CCA) properties were measured using a Mylab90 platform (Esaote, Genova, Italy) equipped with a linear 5-13 MHz transducer (LA523) and a previously validated radiofrequency-based tracking system that allowed the real-time determination of carotid far-wall intima-media thickness (Quality Intima-Media Thickness), diameter, and distension (Quality Arterial Stiffness) with a high spatial and temporal resolution $(9,10)$.

\section{Cardiac and peripheral artery involvement}

A 12-lead electrocardiogram was recorded with standard equipment after resting for at least 5 minutes. Transthoracic echocardiography was performed using commercially available equipment (Vivid I; GE Vingmed Ultrasound, Horten, Norway). Ultrasonographic measurements of structural and functional parameters were conducted according to the Guideline of the American Society of Echocardiography (11-13).

Bilateral ankle brachial index was used to evaluate the peripheral artery involvement. BaPWV was assessed automatically in the supine position using an oscillometric method (VP-1000, Colin, Komaki, Japan) (14). 


\section{Assessment of motor performance}

Motor functions were evaluated using a series of motor function tests, which were widely used in previous studies of community dwelling individuals, patients with cerebral small vessel disease, and those with Parkinson's disease. The extensive test batteries that assessed both the lower and upper extremities included the Short Physical Performance Battery (15), Assessment and Rating of Ataxia (16), Tinetti Performance-Oriented Assessment of Mobility (17), Timed Up and Go test (18), and Unified Parkinson's Disease Rating Scale (19). To evaluate gait speed, participants were asked to walk a distance of 3 meters at their usual pace. All motor function evaluations were performed in front of a self-constructed Kinect-based system, which automatically recognized the target examinee, extracted the walking segmentations, and acquired quantitative gait parameters using a built-in movement detection model (20). Using this system, we collected in-depth videos of the examinee's posture and limb movement and quantified the examinee's motor function.

\section{Assessment of cognition and activities of daily living}

Comprehensive neuropsychological (NP) assessments were conducted to measure global cognition and investigate cognitive domains of memory, language, attention, visuospatial perception, and executive functions, comparable to those of other known research cohorts (21-23). The Chinese versions of the Mini-Mental State Examination (C-MMSE) and Montreal Cognitive Assessment were used as screening tools $(24,25)$. A set of NP tests was administered to all participants who gave consent, which included the Chinese versions of Fuld Object Memory Test, Rapid Verbal Retrieval Test, Block Design, Digit Span, and the Trail Making Test (26). When participants' MMSE score was $\leq 24$ or complaints of cognitive difficulties were raised, additional assessments were undertaken using the Clinical Dementia Rating Scale $(27,28)$. All participants were administered the Hamilton Rating Scale to screen for depression (29). As a measure of disability, the Barthel Index was used (30). The activities of daily living and health-related quality of life were assessed by the Alzheimer's Disease Cooperative StudyActivities of Daily Living scale (31) and the Short Form Health Survey 36 (32).

In the process of cognitive assessments, evaluation of handwriting function was conducted on a Wacom pen display using a stylus with a sampling frequency of $100 \mathrm{~Hz}(33)$.
The screen size was 13.3 inches, with a resolution of $1,280 \times 800$ pixels, resulting in a pixel pitch of $0.224 \mathrm{~mm}$. The device collected time series of sampled data during drawing. Each sample point included the $\mathrm{x}$ and $\mathrm{y}$ coordinates, pressure, tilt, orientation, and self-rotation of the pen. Statistical, time-domain, frequency-domain, and entropy methods were used to extract indicators from the data and evaluate the examinee's motor and cognitive functions.

\section{Definitions}

Hypertension was defined as self-reported hypertension, treatment with antihypertensive medication, systolic blood pressure $\geq 140 \mathrm{mmHg}$, or diastolic blood pressure $\geq 90 \mathrm{mmHg}$. Diabetes mellitus was defined as selfreported diabetes, use of oral antidiabetic drugs or insulin, fasting serum glucose $\geq 7.0 \mathrm{mmol} / \mathrm{L}$, or hemoglobin A1c $\geq 6.5 \%$. Hyperlipidemia was defined as fasting serum total cholesterol $>5.2 \mathrm{mmol} / \mathrm{L}$, low density lipoprotein cholesterol $>3.62 \mathrm{mmol} / \mathrm{L}$ or use of lipid-lowering drugs. Smoking status was classified as current smoker (at least within the prior month) or non-current smoker.

\section{Annual monitoring and long-term follow-up}

The cohort has been followed up annually since 2017 via on-site interviews. For participants who reported the occurrence of vascular events (myocardial infarction, unstable angina, and stroke) during follow-up, further information was obtained from their medical records. Information of mortality in Shunyi district was reported annually using the district's death registration system. In addition, health-related data, measures of major cardiovascular risk factors, neurological examinations, and fresh biological samples were collected annually. Cognitive assessments were followed up every 3 years. Since 2019, a follow-up brain MRI is being performed using the same scanner. The measures at baseline visits and follow-up visits are summarized in Table 2.

\section{Statistical analysis}

Demographics and participant characteristics are presented using descriptive statistical analyses. Comparison of data between groups was conducted using the $t$-test (for means), Wilcoxon rank-sum test (for medians), and chi-square test (for percentages). The associations between baseline characteristics and outcomes were assessed by multivariable 
Table 2 Summary of measures at baseline and follow-up in the Shunyi Study

\begin{tabular}{|c|c|c|c|c|}
\hline \multirow{3}{*}{ Measure } & \multicolumn{4}{|c|}{ Time-points } \\
\hline & \multirow{2}{*}{$\begin{array}{l}\text { Baseline } \\
\text { Jun. 2013- } \\
\text { Sep. } 2014\end{array}$} & \multicolumn{3}{|c|}{ Follow-up } \\
\hline & & $\begin{array}{l}\text { Jul.-Sep. } \\
\quad 2017\end{array}$ & $\begin{array}{l}\text { Jul.-Sep. } \\
2018\end{array}$ & $\begin{array}{l}\text { Jul.-Sep. } \\
2019\end{array}$ \\
\hline Consent form & $\bullet$ & $\bullet$ & $\bullet$ & $\bullet$ \\
\hline $\begin{array}{l}\text { Follow-up questionnaire (including newly diagnosed cardiovascular or cerebral } \\
\text { vascular events, kidney disease, hypertension and diabetes) }\end{array}$ & & $\bullet$ & $\bullet$ & $\bullet$ \\
\hline $\begin{array}{l}\text { Anthropometric measurement (body height, body weight, body mass index, } \\
\text { waist circumference and hip circumference) }\end{array}$ & $\bullet$ & $\bullet$ & $\bullet$ & $\bullet$ \\
\hline $\begin{array}{l}\text { Venous blood biochemical parameters (blood glucose, hemoglobin A1C, } \\
\text { blood lipid profile, serum creatinine, uric acid, electrolytes, homocysteine, } \\
\text { thyroid function, etc.) }\end{array}$ & $\bullet$ & $\bullet$ & $\bullet$ & $\bullet$ \\
\hline Urinalysis (urine microalbumin, urine creatinine) & $\bullet$ & $\bullet$ & $\bullet$ & $\bullet$ \\
\hline Blood and urine sample collection & $\bullet$ & $\bullet$ & $\bullet$ & $\bullet$ \\
\hline Whole exome sequencing & $\bullet$ & & & \\
\hline Brain magnetic resonance imaging ${ }^{\dagger}$ & $\bullet$ & & & $\bullet$ \\
\hline Carotid ultrasonography & $\bullet$ & & & $\bullet$ \\
\hline Major cardiovascular events & & $\bullet$ & $\bullet$ & • \\
\hline All-cause deaths & & • & - & - \\
\hline
\end{tabular}

regression models.

\section{Results}

The baseline characteristics of the Shunyi study population are presented in Table 3. Among the 1,586 participants included, $40 \%$ were men, and the mean age of the population was $56.7 \pm 10.0$ years. The proportion of illiterate participants was $9.5 \%$, and only $1.3 \%$ of the population had a college-level or higher education. The proportion of current smokers was $24.0 \%$ (men: $56.9 \%$, women: $2.1 \%$ ). The prevalence of major vascular risk factors at baseline was $52.5 \%$ for hypertension, $17.3 \%$ for diabetes mellitus, and $46.1 \%$ for hyperlipidemia. Six percent of the study participants reported a history of stroke. Thirty-nine percent of the participants used at least one medication for vascular diseases, mainly antihypertensive drugs.

The cohort has been followed up annually since 2017 . As of October 2019, we had failed to obtain follow-up data of five participants because they migrated. The final results 
Table 3 Baseline characteristics of the study population

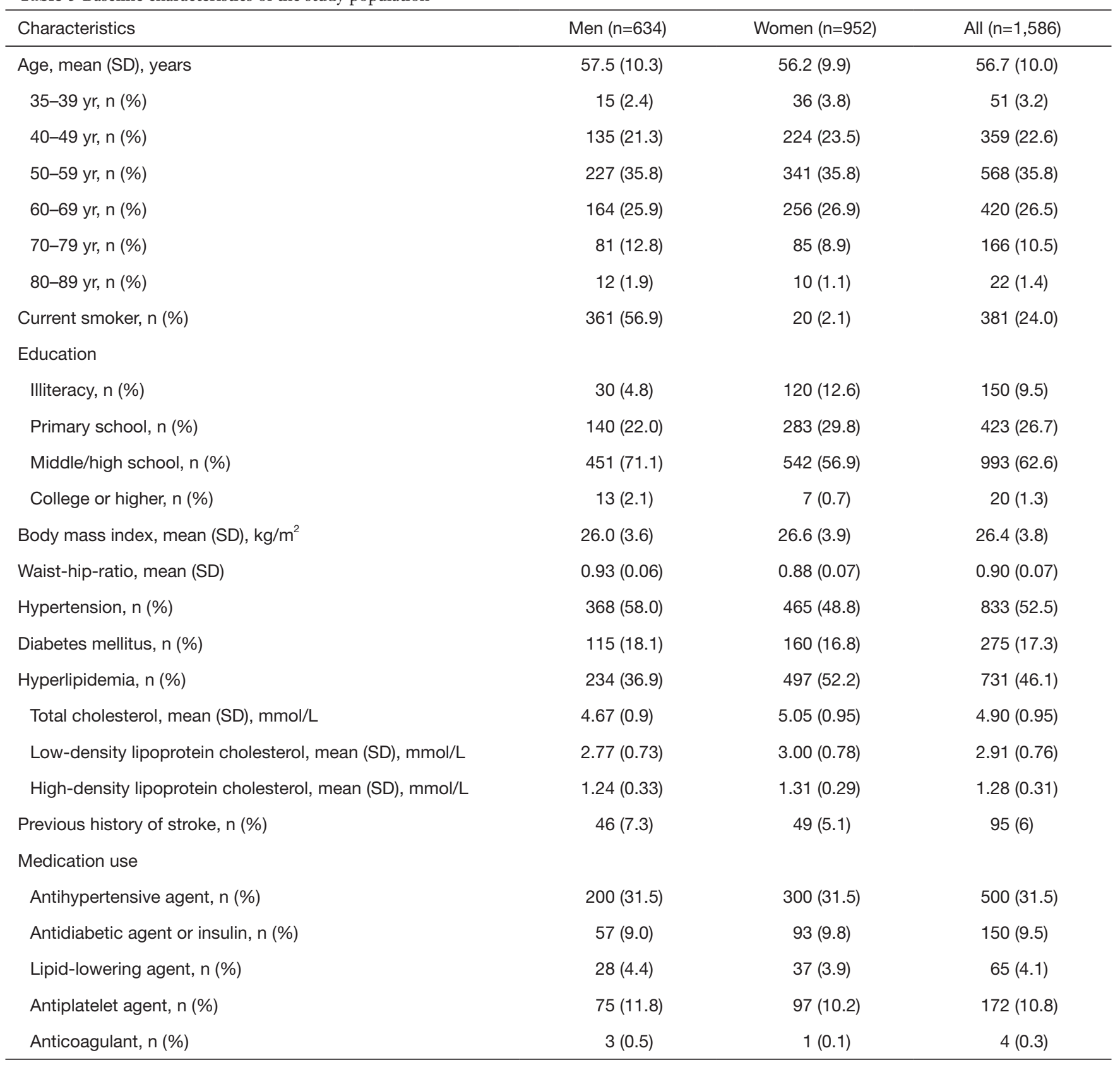

SD, standard deviation.

of this study will be published in peer-reviewed medical journals.

\section{Discussion}

The Shunyi cohort study is a community-based cohort study with a study design similar to that of the Framingham Heart
Study $(5,6)$ and the Atherosclerosis Risk in Communities Study (34) conducted in the United States, the Rotterdam Study $(4,35)$ conducted in the Netherlands, and the Age, Gene/Environment Susceptibility-Reykjavik Study (36) conducted in Iceland. Considering the comprehensive data collected and continued clinical surveillance, the Shunyi study targets cardiovascular diseases, neurological diseases 
and age-related brain changes, endocrine disorders, and locomotor diseases. Additionally, the Shunyi study provides an opportunity to compare data of Chinese individuals with those of cohorts belonging to other races and ethnicities. The study design and rationale are presented herein.

The mean age of the participants in the Shunyi cohort was 56.7 years, younger than that of the Framingham Heart Study and Rotterdam Study. Brain MRI research has reported the prevalence of imaging markers of cerebral small vessel disease in this community (37). On comparing participants in the same age group to those from regions of the Western world, a higher burden of cerebral small vessel disease, such as lacunes, was observed in the Shunyi cohort (37). Furthermore, on baseline MRI data analysis, $3.6 \%(45 / 1,257)$ of the participants experienced at least one episode of stroke during a mean follow-up of 4.3 years in the Shunyi study. By contrast, in the Rotterdam Study, only $2.0 \%(93 / 4,759)$ of participants experienced an episode of stroke over a mean follow-up of 4.9 years (38). These findings might be explained by the lifestyle and sociodemographic changes that may have had an impact on the prevalence of common vascular risk factors. The annual income per person among the rural residents of Shunyi district was 19,629 Chinese Yuan (CNY) in 2014, while that of residents in urban Beijing was 44,489 CNY (39). When considering education level, as listed in Table 3, less than $2 \%$ of the Shunyi population had a college-level or higher education, compared with $65 \%$ of the sample in the Framingham Heart Study (40). A relatively heavy burden of vascular risk factors with a lower control rate was also observed in the Shunyi population. The prevalence of hypertension was as high as $52.5 \%$ at baseline, whereas the rate of use of antihypertensive agents was only $31.5 \%$. In contrast, more than $80 \%$ of the hypertensive subjects in the Framingham Heart Study were taking antihypertensive medications (40). From these data, one can discern that this community is a suitable representative sample in this period of China's economic transition. Although the living standard has increased, education level and health literacy awareness are still low (41). These characteristics of this population provide insights into the determinants and consequences of cardiovascular disease and age-related brain alterations, which are different from those in developed countries.

Along with the classic evaluation methods, innovative techniques have been applied in this cohort. Motor function was quantitatively evaluated by a Kinect-based system and automatic gait segmentation. In addition, handwriting function was quantitatively evaluated by the Wacom pen display and stylus during cognitive tests. These data are valuable for a longitudinal study, as changes of motor function over time can be quantified. Furthermore, we performed whole-exome sequencing and genotyping with ASA BeadChip simultaneously to investigate both common and rare mutations linked to age-related diseases in this population. Other new techniques, which are rarely applied in other population-based cohorts, include whole-body T1 Dixon imaging by $3 \mathrm{~T}$ MRI, and CCA stiffness measurement by carotid ultrasonography. We believe that these innovative techniques can provide new research prospects.

The major strength of this study is its populationbased prospective design. Other strengths include detailed baseline information, well-stored biological specimens, full spectrum of MRI scans with high resolution and sensitivity to evaluate structural and vascular changes in the brain, comprehensive and quantitative assessments of cognitive and motor functions, and active surveillance for illnesses with high compliance. In addition, whole-exome sequencing and chip were used to portray the genetic characteristics of this population, making hereditary research possible. The well-established protocol holds an extensive range of data, and all clinical, biological, and imaging measures will be followed up continuously.

There are some limitations that need to be addressed. Individuals who refused to participate or those with MRI contraindications in this cohort were generally older and less healthy than those with available MRI data, leading to a possibility of selection bias. This study focused on a population in rural Beijing, a district in economic transition, composed of a large proportion of individuals with less education and a relatively higher prevalence but lower control rates of vascular risk factors. These factors may limit the generalizability of our conclusions to some extent.

\section{Conclusions}

With an extensive range of clinical, genetic, and imaging data, the Shunyi cohort study has the potential to make significant contributions towards unraveling the causes and consequences of cardiovascular diseases and age-related brain changes.

\section{Acknowledgments}

Funding: This work was supported by National Key 
Research and Development Program of China (grant numbers: 2016YFB1001402, 2016YFC1300505, 2017YFE0100500), National Key Technology R\&D Program in the 12 th five-year Plan of China (grant numbers: 2012BAJ18B04-3, 2015AA020506), National Natural Science Foundation of China (grant numbers: 81671173, 81173663), Strategic Priority Research Program (Pilot study) "Biological basis of aging and therapeutic strategies" of the Chinese Academy of Sciences (grant number: XDPB10), the CAMS Innovation Fund for Medical Sciences (grant number: CIFMS \#2017-I2M-3-008), and Research Foundation for Young Scholars of Peking Union Medical College Hospital (PUMCH201911275).

\section{Footnote}

Reporting Checklist: The authors have completed the STROBE reporting checklist. Available at http://dx.doi. org/10.21037/atm-20-4195

Data Sharing Statement: Available at http://dx.doi. org/10.21037/atm-20-4195

Peer Review File: Available at http://dx.doi.org/10.21037/ atm-20-4195

Conflicts of Interest: All authors have completed the ICMJE uniform disclosure form (available at http://dx.doi. org/10.21037/atm-20-4195). The authors have no conflicts of interest to declare.

Ethical Statement: The authors are accountable for all aspects of the work in ensuring that questions related to the accuracy or integrity of any part of the work are appropriately investigated and resolved. This study was conducted in accordance with the Declaration of Helsinki (as revised in 2013). The study was approved by the Ethical Committee of Peking Union Medical College Hospital (No: B-160), and informed consent was taken from all the participants.

Open Access Statement: This is an Open Access article distributed in accordance with the Creative Commons Attribution-NonCommercial-NoDerivs 4.0 International License (CC BY-NC-ND 4.0), which permits the noncommercial replication and distribution of the article with the strict proviso that no changes or edits are made and the original work is properly cited (including links to both the formal publication through the relevant DOI and the license). See: https://creativecommons.org/licenses/by-nc-nd/4.0/.

\section{References}

1. Yang G, Wang $Y$, Zeng $Y$, et al. Rapid health transition in China, 1990-2010: findings from the Global Burden of Disease Study 2010. Lancet 2013;381:1987-2015.

2. Yang G, Kong L, Zhao W, et al. Emergence of chronic non-communicable diseases in China. Lancet 2008;372:1697-705.

3. Prince MJ, Wu F, Guo Y, et al. The burden of disease in older people and implications for health policy and practice. Lancet 2015;385:549-62.

4. Ikram MA, Brusselle GGO, Murad SD, et al. The Rotterdam Study: 2018 update on objectives, design and main results. Eur J Epidemiol 2017;32:807-50.

5. Chen G, Levy D. Contributions of the Framingham Heart Study to the Epidemiology of Coronary Heart Disease. JAMA Cardiol 2016;1:825-30.

6. Wolf PA. Contributions of the Framingham Heart Study to stroke and dementia epidemiologic research at 60 years. Arch Neurol 2012;69:567-71.

7. Chauhan G, Adams HHH, Satizabal CL, et al. Genetic and lifestyle risk factors for MRI-defined brain infarcts in a population-based setting. Neurology 2019;92:e486-e503.

8. Li L, Lyu J. Large prospective cohort studies: a review and update. Zhonghua Liu Xing Bing Xue Za Zhi 2015;36:1187-9.

9. Palombo C, Kozakova M, Guraschi N, et al. Radiofrequency-based carotid wall tracking: a comparison between two different systems. J Hypertens 2012;30:1614-9.

10. Meinders JM, Hoeks AP. Simultaneous assessment of diameter and pressure waveforms in the carotid artery. Ultrasound Med Biol 2004;30:147-54.

11. Yongtai L, Jinzhi L, Lixin Z, et al. Effect of different ranges of systolic blood pressure on left ventricular structure and diastolic function in a Chinese population: a cross-sectional population-based Shunyi study. BMJ Open 2019;9:e028398.

12. Lang RM, Badano LP, Mor-Avi V, et al. Recommendations for cardiac chamber quantification by echocardiography in adults: an update from the American Society of Echocardiography and the European Association of Cardiovascular Imaging. J Am Soc Echocardiogr 
2015;28:1-39.e14.

13. Nagueh SF, Smiseth OA, Appleton CP, et al.

Recommendations for the Evaluation of Left Ventricular Diastolic Function by Echocardiography: An Update from the American Society of Echocardiography and the European Association of Cardiovascular Imaging. J Am Soc Echocardiogr 2016;29:277-314.

14. Yamashina A, Tomiyama H, Takeda K, et al. Validity, reproducibility, and clinical significance of noninvasive brachial ankle pulse wave velocity measurement. Hypertens Res 2002;25:359-64.

15. Guralnik JM, Simonsick EM, Ferrucci L, et al. A short physical performance battery assessing lower extremity function: association with self-reported disability and prediction of mortality and nursing home admission. J Gerontol 1994;49:M85-94.

16. Schmitz-Hübsch T, du Montcel ST, Baliko L, et al. Scale for the assessment and rating of ataxia: development of a new clinical scale. Neurology 2006;66:1717-20.

17. Tinetti ME. Performance-oriented assessment of mobility problems in elderly patients. J Am Geriatr Soc 1986;34:119-26.

18. Podsiadlo D, Richardson S. The Timed "Up \& Go": a test of basic functional mobility for frail elderly persons. J Am Geriatr Soc 1991;39:142-8.

19. Goetz CG, Tilley BC, Shaftman SR, et al. Movement Disorder Society-sponsored revision of the Unified Parkinson's Disease Rating Scale (MDS-UPDRS): scale presentation and clinimetric testing results. Mov Disord 2008;23:2129-70.

20. Z Zhang. Microsoft Kinect Sensor and Its Effect. IEEE MultiMedia 2012;19:4-10.

21. de Groot JC, de Leeuw FE, Oudkerk M, et al. Cerebral white matter lesions and cognitive function: the Rotterdam Scan Study. Ann Neurol 2000;47:145-51.

22. 3C Study Group. Vascular factors and risk of dementia: design of the Three-City Study and baseline characteristics of the study population. Neuroepidemiology 2003;22:316-25.

23. van Norden AG, de Laat KF, Gons RA, et al. Causes and consequences of cerebral small vessel disease. The RUN DMC study: a prospective cohort study. Study rationale and protocol. BMC Neurol 2011;11:29.

24. Zhang ZX, Hong X, Li H, et al. The mini-mental state examination in the Chinese residents population aged 55 years and over in the urban and rural areas of Beijing (in Chinese). Chinese Journal of Neurology 1999;32:149-53.
25. Wen HB, Zhang ZX, Niu FS, et al. The application of Montreal cognitive assessment in urban Chinese residents of Beijing (in Chinese). Chinese Journal of Internal Medicine 2008;47:36-9.

26. Zhang M, Katzman R, Yu E, et al. A preliminary analysis of incidence of dementia in Shanghai, China. Psychiatry Clin Neurosci 1998;52:S291-4.

27. Morris JC. The Clinical Dementia Rating (CDR): Current version and scoring rules. Neurology 1993;43:2412-4.

28. Lim WS, Chong MS, Sahadevan S. Utility of the clinical dementia rating in Asian populations. Clin Med Res 2007;5:61-70.

29. Hamilton M. A rating scale for depression. J Neurol Neurosurg Psychiatry 1960;23:56-62.

30. Leung SO, Chan CC, Shah S. Development of a Chinese version of the Modified Barthel Index: validity and reliability. Clin Rehabil 2007;21:912-22.

31. Galasko D, Bennett D, Sano M, et al. An inventory to assess activities of daily living for clinical trials in Alzheimer's disease. The Alzheimer's Disease Cooperative Study. Alzheimer Dis Assoc Disord 1997;11:S33-9.

32. Ware JE Jr, Sherbourne CD. The MOS 36-item shortform health survey (SF-36). I. Conceptual framework and item selection. Med Care 1992;30:473-83.

33. Wacom. 2019. Interactive Pen Displays \& Touch Screen Tablets I Wacom. Available online: http://www.wacom. com/en-us/products/pen-displays

34. The ARIC Investigators. The Atherosclerosis Risk in Communities (ARIC) Study: Design and Objectives. Am J Epidemiol 1989;129:687-702.

35. Hofman A, Grobbee DE, de Jong PT, et al. Determinants of disease and disability in the elderly: the Rotterdam Elderly Study. Eur J Epidemiol 1991;7:403-22.

36. Muller M, Sigurdsson S, Kjartansson O, et al. Late-life Brain Volume: A Life-Course Approach. The AGESReykjavik Study. Neurobiol Aging 2016;41:86-92.

37. Han F, Zhai FF, Wang Q, et al. Prevalence and Risk Factors of Cerebral Small Vessel Disease in a Chinese Population-Based Sample. J Stroke2018;20:239-46.

38. Akoudad S, Portegies ML, Koudstaal PJ, et al. Cerebral Microbleeds Are Associated with an Increased Risk of Stroke: The Rotterdam Study. Circulation 2015;132:509-16.

39. Beijing Government Network. Beijing regional statistical yearbook 2015. Available online: http://202.96.40.155/nj/ qxnj/2015/zk/indexce.htm

40. Romero JR, Beiser A, Himali JJ, et al. Cerebral 
Microbleeds and risk of Incident Dementia: The

Framingham Heart Study. Neurobiol Aging 2017;54:94-9. 41. Zhao YY, He C, Zheng QG. Analysis of Health

Cite this article as: Han F, Zhou LX, Ni J, Yao M, Zhai FF, Liu YT, Wu W, Xue HD, Li ML, Yang M, Dai Q, Cui LY, Jin ZY, Zhu YC, Zhang SY. Design of the Shunyi study on cardiovascular disease and age-related brain changes: a community-based, prospective, cohort study. Ann Transl Med 2020;8(23):1579. doi: 10.21037/atm-20-4195
Knowledge Awareness and Health Needs Investigation among Rural Residents in Shunyi District of Beijing City (in Chinese). Occup and Health 2010;26:2962-4. 